EPSC Abstracts

Vol., EPSC2020-592, 2020

Europlanet Science Congress 2020

(C) Author(s) 2020. This work is distributed under

the Creative Commons Attribution 4.0 License.

\title{
A dynamic study of the post-impact transport of Lunar rocks to Earth and its application to the Sept. 11th, 2013 impact
}

\author{
Esther Mas-Sanz ${ }^{1,2}$, Josep M. Trigo-Rodríguez ${ }^{1,3}$, and Eloy Peña-Asensio ${ }^{1,4}$ \\ ${ }^{1}$ Institut d’Estudis Espacials de Catalunya (IEEC), Gran Capità 2-4, Ed. Nexus, desp. 201, 08034 Barcelona, Catalonia, Spain \\ ${ }^{2}$ ESEIAAT, Universitat Politècnica de Catalunya (UPC), 08222 Terrassa, Catalonia, Spain \\ ${ }^{3}$ Institute of Space Sciences (CSIC), Meteorites, Minor Bodies and Planetary Sciences Group, 08193 Cerdanyola del Vallès, \\ Barcelona, Catalonia, Spain (trigo@ice.csic.es) \\ ${ }^{4}$ Universitat Autònoma de Barcelona (UAB), 08193 Bellaterra, Barcelona, Catalonia, Spain
}

\section{Introduction}

Lunar meteorites constitute direct, varied samples of the materials forming the lunar surface, allowing to study its bulk chemistry, mineralogy and even geological history. The study of these meteorites not only sheds light on the composition of the Moon but also on its formation and the origin of the Solar System itself. Nowadays we know that these Lunar rocks are delivered to Earth in relative short timescales after being ejected by the impact of an asteroid or a comet from the lunar surface. So far, no lunar meteorite has ever been associated with a crater, not even has been witnessed to fall on Earth [1].

The atmosphere of the Moon is extremely thin, being unable to decelerate particles that are either impacting or escaping from the Moon [2]. It is due to the impact of these high-velocity projectiles that some lunar rocks can be ejected with a velocity above the escape velocity of the Moon (2.38 $\mathrm{km} / \mathrm{s}$ ) [3]. When a projectile of a certain mass impacts the surface of the Moon excavates an impact crater and releases a mass between 3-4 orders of magnitude higher than the projectile mass [3,4]. Most of the ejected mass falls near the crater and forms continuous ejecta blankets, while the rest follows an impact trajectory far away from the initial impact, while a small part of the ejecta achieves enough velocity to escape the Moon [3,4]. Our study focuses on the dynamic evolution of these Lunar rocks once they left our satellite, and their ability to reach the Earth as meteorites.

\section{Methodology}

This study simulates trajectories of lunar ejecta to explore which parameters influence on their fate (impactor/survivor) and their transfer time using the Mercury 6 software [5]. It has been built upon the results and possible time limitations of a previous study [6]. Ejecta trajectories are calculated with a simplified Solar System model (Earth-Moon-Sun) when time scales are below 1000 years (short transfers). Beyond this value (long transfers, up to 100,000 years), secular perturbations caused by near-by planets are no longer negligible [7] and an 8-planet Solar System model needs to be implemented. Particles found in the vicinity of Earth, at a maximum of 10 Earth's Hill radii, are considered to be in geocentric orbit while those beyond belong to heliocentric orbits. The parameters changed in each simulation were: the local azimuth angle and elevation, launch velocity and the 
date of the launch. Additionally, the orbital parameters compiled and studied to understand the ejecta evolution and delivery time to our planet.

We have also analyzed a case study corresponding with the impact flare recorded on Sept. $11^{\text {th }}$,

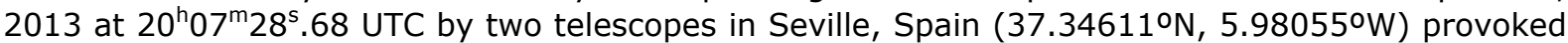
by the impact of a meteoroid on the surface of the Moon [8]. The impact was located in the west region of the Mare Nubium, at $17.2^{\circ} \mathrm{S} 20.5^{\circ} \mathrm{W}$ (selenographic coordinates). We performed a simulation with 360 particles launched across an arc of $360^{\circ}$ from the Mare Nubium impact point, ejected at $45^{\circ}$ with respect to the local vertical and with a launch velocity $2.6 \mathrm{~km} / \mathrm{s}$. The particle's evolution was then propagated until $10^{4}$ years to get information about the delivery efficiency, and common timescale.

\section{Results}

In general we noticed that most ejecta are reaching the Earth in particularly short timescales, between a few days and 10 years. For this fast transfers, predicting if the ejecta will become a future impactor depends only on the launch angle and ejection velocity. Parameters at launch have less and less influence on the fate of the ejecta as time increases. For delivery timescales larger than 1000 years any particle may collide with Earth regardless of its launch angle. The evolution of the main orbital elements for a typical long case is described in Fig. 1. The peak of accumulated collisions shifts to a lower launch velocity at $2.4 \mathrm{~km} / \mathrm{s}$.
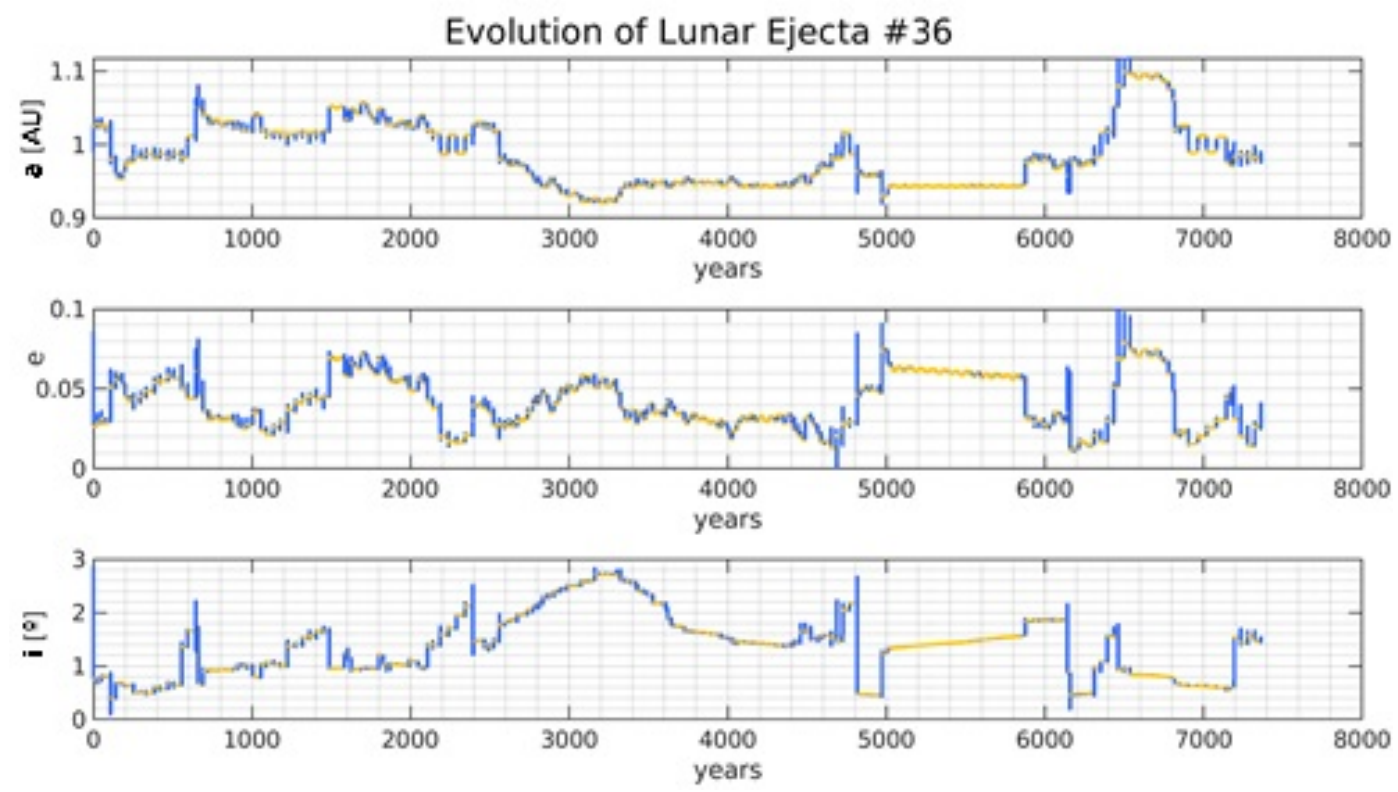

Figure 1. Evolution of a lunar ejecta impacting with Earth in 7500 years. Stability periods correspond to heliocentric stages (yellow), perturbations occur in geocentric stage (blue).

In general, the orbital elements $(a, e, i)$ present higher oscillations during geocentric orbits than during the heliocentric orbit stages. However, the orbital elements of survivor particles and those of the impactors remain indistinguishable before the impact event.

Concerning the impact event occurred on Sept. $11^{\text {th }}, 2013$ our simulation shows that most rocks reach Earth is relatively short timescales (Fig. 2). A significant number of rocks reach our planet during the first decade after the impact. As a future work we wish to quantify the geometric entry circumstances and the geocentric velocity for selected cases to get clues to better identify these rare fireballs among the heliocentric population. 


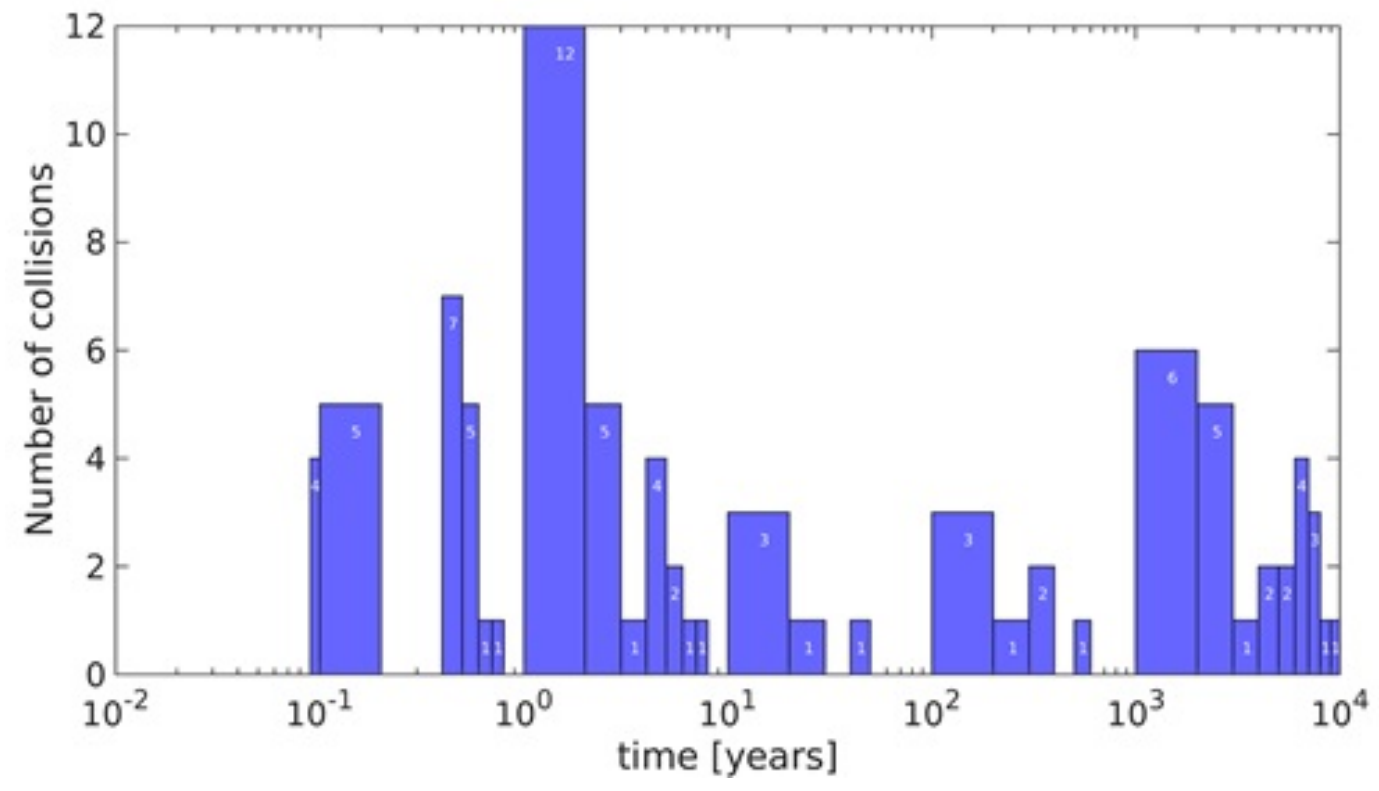

Figure 2. Sept. 11th, 2013 simulation: number of collisions with Earth over time.

\section{Conclusions}

The parameters that influence on the fate of the Lunar ejecta (short transfers) are:

- Launch velocity: accumulated collisions with Earth reach a peak for velocities between 2.6 and $2.7 \mathrm{~km} / \mathrm{s}$. For these values nearly $20 \%$ of the original ejecta population has impacted with Earth in the first 100 years.

- Launch angle (relative angle between launch velocity and lunar velocity): This angle results from the combination of local azimuth, local elevation and particular launch site. When its value is $\approx 120^{\circ}$ or above ejecta escape the Moon's gravity with barely any velocity left and eventually "fall" due to Earth's pull.

- Ejection angle: default is considered at $45^{\circ}$. Its variation only influences Earth collisions for peak collision velocities (between 2.6 and $2.7 \mathrm{~km} / \mathrm{s}$ ) when launches are towards the East or the West (in selenographic coordinates).

\section{Acknowledgements}

The authors acknowledge financial support from the Spanish Ministry (PGC2018-097374-B-I00, PI: JMTR).

\section{References}

[1] Korotev R. L. (2005) Chemie der Erde 65, 297-346.

[2] Fritz, J. (2012) Icarus. 221, 1183-1186.

[3] Artemieva, N. A. and Shuvalov, V. V., (2008) Solar System Res. 42, 329-334.

[4] Melosh, H. J.,, Oxford University Press, 1989.

[5] Chambers, J. E. (1999), MNRAS 304, 793-799.

[6] Gladman, B. J., PhD thesis, 1996.

[7] Galibina, I. V. and Terenteva (1982), A. K., AVest , 15, 132-137 
[8] Madiedo, J. M. et al. (2015) MNRAS 439, 2364- 2369. 